\title{
VECTOR FIELDS, RESIDUES AND COHOMOLOGY
}

\author{
JAMES B. CARRELL \\ Department of Mathematics, University of British Columbia, \\ 121-1984 Mathematics Road, Vancouver, B.C. V6T 1Z2, Canada \\ E-mail:carrell@math.ubc.ca
}

1. Residue formulas. Let $E$ be a holomorphic vector bundle on a compact complex manifold $X$ of dimension $n$ with structure sheaf $\mathcal{O}_{X}$, and let $\mathcal{E}$ be the locally free sheaf of $\mathcal{O}_{X}$-modules (or briefly, an $\mathcal{O}_{X}$-sheaf) canonically associated to $E$. A residue formula for $E$ expresses the Chern numbers of $E$ as finite sums of residues. Recall that a Chern number is associated to a symmetric $\mathcal{O}_{X}$-linear map $p: \operatorname{End}_{\mathcal{O}_{X}}(\mathcal{E})^{\otimes n} \rightarrow \mathcal{O}_{X}$ as follows. Letting $\left.\tilde{c}(E) \in H^{1}\left(X, \operatorname{End}_{\mathcal{O}_{X}}(\mathcal{E}) \otimes \Omega_{X}^{1}\right)\right)$ denote the Chern class of $E$ in the sense of [At], one may apply $p$ to $\tilde{c}(E)$ to obtain a class $p(E)=p(\tilde{c}(E)) \in H^{n}\left(X, \Omega_{X}^{n}\right)$ which may be evaluated on the fundamental cycle of $X$. The number $(2 \pi i)^{-n} \int_{X} p(E)$ is the associated Chern number of $X$; we will discuss computing these numbers as sums of residues.

Let $\Theta_{X}$ denote the sheaf of sections of the holomorphic tangent bundle $W$ of $X$. Let $\mathcal{L}$ be an invertible $\mathcal{O}_{X}$-sheaf, and assume $V \in H^{0}\left(X, \Theta_{X} \otimes \mathcal{L}\right)$ is a section that has only isolated zeros. The zero set of $V$ can be given the structure of a possibly unreduced scheme $Z$, called the zero scheme of $V$. Namely, $Z$ is the finite subscheme of $X$ defined by the sheaf of ideals $\mathcal{I}_{Z}=i(V)\left(\Omega_{X}^{1} \otimes \mathcal{L}^{-1}\right) \subset \mathcal{O}_{X}$, where, $i(V): \Omega_{X}^{1} \otimes \mathcal{L}^{-1} \rightarrow \mathcal{O}_{X}$ denotes the canonical contraction operator defined by viewing $V$ as an operator $V: \Omega_{X}^{1} \rightarrow \mathcal{L}$ (so $i(V)=V \otimes 1)$. The structure sheaf of $Z$ is denoted by $\mathcal{O}_{Z}$. Thus $\mathcal{O}_{Z}:=\mathcal{O}_{X} / \mathcal{I}_{Z}$.

Letting $\mathcal{L}_{Z}:=\mathcal{L} \otimes \mathcal{O}_{Z}$ denote the pull back to $Z$ of $\mathcal{L}$, there is a canonical $\mathbb{C}$-linear map $\operatorname{Res}_{V}: H^{0}\left(Z, \mathcal{L}_{Z}^{n}\right) \rightarrow \mathbb{C}$ called the Grothendieck residue morphism [C1], [CL2], which is based on [Be], [H], [V] (also see [L]). A residue formula for a pair $(p, E)$ as above will consist of using $V$ to associate a natural class $p_{Z}(E) \in H^{0}\left(Z, \mathcal{L}_{Z}^{n}\right)$ to $p(E)$ (the localization to $Z$ ) such that

$$
(2 \pi i)^{-n} \int_{X} p(E)=\operatorname{Res}_{V}\left(p_{Z}(E)\right),
$$

where $\mathcal{L}, V, Z$ are as above.

1991 Mathematics Subject Classification: Primary: 32C10, 32J27. Secondary: 32A27, 32S65, 14M15, 14L30.

Partially supported by a grant of the Natural Sciences and Engineering research Council of Canada.

The paper is in final form and no version of it will be published elsewhere. 
The first general residue formula of this type is the celebrated theorem of Bott [B1]. Recall that if $V \in H^{0}\left(X, \Theta_{X}\right)$ is a holomorphic vector field on $X$, an isolated zero $\zeta$ of $V$ is called simple if the element $A(\zeta) \in \operatorname{End}\left(W_{\zeta}\right)$ induced by the Lie derivative on $\Theta_{X}$ with respect to $V$ is an isomorphism.

THEOREM 1. Let $X$ be a compact complex manifold of dimension $n$ and $V \in H^{0}\left(X, \Theta_{X}\right)$ a holomorphic vector field on $X$ with simple isolated zeros $\zeta_{1}, \ldots, \zeta_{k}$. Then for any symmetric $\mathcal{O}_{X}$-linear map $p: \operatorname{End}_{\mathcal{O}_{X}}(\mathcal{W})^{\otimes n} \rightarrow \mathcal{O}_{X}$, one has

$$
(2 \pi i)^{-n} \int_{X} p(W)=\sum_{i} \frac{p\left(A_{\zeta_{i}}\right)}{\operatorname{Det} A\left(\zeta_{i}\right)} .
$$

In the setting of this theorem, $\mathcal{L}=\mathcal{O}_{X}$, and $Z$ is the set of zeros of $V$, as explained below. If $V$ is non-vanishing, then the right-hand side of (1.2) is zero. When the degree of $p$ is less than $n$, it is also the case that the right-hand side of (1.2) is zero, although this does not follow directly from (1.2) (see Section 4 below).

2. Equivariant sheaves. We will state Bott's theorem in a general form, after introducing the concept of an equivariant sheaf, which is a useful extension of the original definition given in [B2]. Let $\mathcal{F}$ be a locally free sheaf of $\mathcal{O}_{X}$-modules and $V \in H^{0}\left(X, \Theta_{X} \otimes \mathcal{F}\right)$ an $\mathcal{F}$-valued holomorphic vector field on $X . V$ can also be viewed as an $\mathcal{O}_{X}$-module homomorphism $\mathcal{F}^{*} \rightarrow \Theta_{X}$, or dually, an $\mathcal{O}_{X}$-module homomorphism $\Omega_{X}^{1} \rightarrow \mathcal{F}$. Notice that it makes sense to talk about the zeros of an $\mathcal{F}$-valued holomorphic vector field; however if the rank of $\mathcal{F}$ is greater than one, $V$ can have more complicated singularities.

One says that an $\mathcal{O}_{X}$-sheaf $\mathcal{E}$ is $V$-equivariant if there exists a $\mathbb{C}$-linear morphism $\mathcal{V}: \mathcal{E} \rightarrow \mathcal{F} \otimes \mathcal{E}$ such that

$$
\mathcal{V}(f \sigma)=V(f) \sigma+f \mathcal{V}(\sigma)
$$

where $f$ (resp. $\sigma$ ) is a local section of $\mathcal{O}_{X}($ resp. $\mathcal{E})$. The requirement of $(2.1)$ is that $\mathcal{V}$ lifts the derivation $V$ of $\mathcal{O}_{X}$.

If $V$ is an ordinary vector field, i.e. $V \in H^{0}\left(X, \Theta_{X}\right)$, then the tangent sheaf $\Theta_{X}$ and the sheaves of holomorphic $p$-forms $\Omega_{X}^{p}$ for $p \geq 0$ are made equivariant by Lie differentiation, i.e. by putting $\mathcal{V}=L_{V}=d i(V)+i(V) d$. A further example is given by a holomorphic foliation of $X$, i.e. a holomorphic subbundle $F$ of the holomorphic tangent bundle $W$ of $X$ which is closed under the Lie bracket of two local sections. Let $\mathcal{F}$ denote the sheaf of sections of $F$, and consider the exact sequence of locally free $\mathcal{O}_{X}$-sheaves

$$
0 \rightarrow \mathcal{F} \rightarrow \Theta_{X} \stackrel{\pi}{\rightarrow} \mathcal{Q} \rightarrow 0
$$

where $\mathcal{Q}$ is the sheaf of sections of the normal bundle $Q=W / F$. Next, let $V$ denote the identity map $I \in H^{0}\left(\mathcal{F}^{*} \otimes \mathcal{F}\right) \subseteq H^{0}\left(\mathcal{F}^{*} \otimes \Theta_{X}\right)$. Then $\mathcal{Q}$ is equivariant with respect to $V$. In fact, a lift $\mathcal{V}$ of $V$ is defined by putting $\mathcal{V}(q)=\pi\left[V, q^{\vee}\right]$, where $q$ is a local section of $\mathcal{Q}$ and $q^{\vee}$ is any lift of $q$ to $\Theta_{X}$.

Recall that a holomorphic connection on $\mathcal{E}$ is a $\mathbb{C}$-linear operator $\nabla: \mathcal{E} \rightarrow \Omega_{X}^{1} \otimes \mathcal{E}$ satisfying the analog of equation (2.1). The obstruction to the existence of a holomorphic connection is the Atiyah-Chern class $\tilde{c}(\mathcal{E}) \in H^{1}\left(X, \operatorname{End}_{\mathcal{O}_{X}}(\mathcal{E}) \otimes \Omega_{X}^{1}\right)([$ At $])$, i.e. $\nabla$ exists if and only if $\tilde{c}(\mathcal{E})=0$. If $V \in H^{0}\left(X, \Theta_{X} \otimes \mathcal{F}\right)$, define $\delta(V) \in H^{1}\left(X, \operatorname{End}_{\mathcal{O}_{X}}(\mathcal{E}) \otimes \mathcal{F}\right)$ by $\delta(V)=i(V) \tilde{c}(\mathcal{E})$.

Proposition 2.3. Let $\mathcal{E}$ be a locally free sheaf of $\mathcal{O}_{X}$-modules. Then $\mathcal{E}$ is $V$-equivariant if and only if $\delta(V)=0$. 
Proof. Just define $\nabla$ locally and put $\mathcal{V}=i(V) \circ \nabla$.

The main point of introducing equivariance is that when $X$ is projective, any locally free $\mathcal{O}_{X}$-sheaf can be made equivariant. In fact we have

Proposition 2.4. If $X$ is projective and $\mathcal{E}$ is locally free, then there exists an invertible $\mathcal{O}_{X}$-sheaf $\mathcal{L}$ such that $\mathcal{E}$ is equivariant with respect to any section of $H^{0}\left(X, \Theta_{X} \otimes \mathcal{L}\right)$ and such that $\Theta_{X} \otimes \mathcal{L}$ has sections with isolated zeros.

P r o of. When $\mathcal{L}$ is sufficiently positive, $H^{1}\left(X, \operatorname{End}_{\mathcal{O}_{X}}(\mathcal{E}) \otimes \mathcal{L}\right)=0$ and $\Theta_{X} \otimes \mathcal{L}$ will be very ample, hence will have sections in general position.

For another application, let us consider a not necessarily integrable holomorphic subbundle $F$ of $W$. Let $V \in H^{0}\left(X, \Theta_{X} \otimes \mathcal{F}^{*}\right)$ be the associated vector field (see (2.2)), and $i(V): \Omega_{X}^{1} \rightarrow \mathcal{F}^{*}$ the corresponding $\mathcal{O}_{X}$-module surjection.

Proposition 2.5. Assume $V$ is as above and that $\mathcal{E}$ is locally free and $V$-equivariant. Then for any symmetric $\mathcal{O}_{X}$-linear $p: \operatorname{End}_{\mathcal{O}_{X}}(\mathcal{E})^{\otimes k} \rightarrow \mathcal{O}_{X}, p(c(\mathcal{E}))=0$ as long as $k>\operatorname{corank} \mathcal{F}$.

P r o of. Consider the exact sequence of locally free sheaves

$$
0 \rightarrow \operatorname{End}_{\mathcal{O}_{X}}(\mathcal{E}) \otimes \mathcal{K} \rightarrow \operatorname{End}_{\mathcal{O}_{X}}(\mathcal{E}) \otimes \Omega_{X}^{1} \rightarrow \operatorname{End}_{\mathcal{O}_{X}}(\mathcal{E}) \otimes \mathcal{F}^{*} \rightarrow 0
$$

where $\mathcal{K}$ is the kernel of $i(V)$. Considering the $H^{1}$-level of the cohomology exact sequence one sees that $V$-equivariance implies $\tilde{c}(\mathcal{E}) \in H^{1}\left(X, \operatorname{End}_{\mathcal{O}_{X}}(\mathcal{E}) \otimes \mathcal{K}\right)$. Hence if $k>$ corank $\mathcal{F}$, then $\tilde{c}(\mathcal{E})^{\otimes k}=0$, since the rank of $\mathcal{K}$ is the corank of $\mathcal{F}$.

Notice that this result applies to the case where $F$ is an integrable subbundle of $W$. Indeed, in that case one may take $\mathcal{E}=\Theta_{X} / \mathcal{F}$, which was shown above to be equivariant. Thus $p(c(\mathcal{E}))=0$ as long as $k>\operatorname{rank} \mathcal{E}$. This is the holomorphic version of a vanishing theorem of Bott [B3].

3. A complex. Assume $V \in H^{0}\left(X, \Theta_{X} \otimes \mathcal{L}\right)$, where $\mathcal{L}$ is an invertible $\mathcal{O}_{X}$-sheaf on $X$. Consider the complex of $\mathcal{O}_{X}$-sheaves

$$
0 \rightarrow \Omega_{X}^{n} \rightarrow \Omega_{X}^{n-1} \otimes \mathcal{L} \rightarrow \cdots \rightarrow \Omega_{X}^{1} \otimes \mathcal{L}^{n-1} \rightarrow \mathcal{L}^{n} \rightarrow 0
$$

with differential $i(V)$ of degree -1 . For a fixed Leray cover $\mathcal{U}$ of $X$, let $\left(K^{\bullet}, D\right)$ denote the complex with

$$
K^{r}=\bigoplus_{q-p=r} C^{q}\left(\mathcal{U}, \Omega_{X}^{p} \otimes \mathcal{L}^{n-p}\right)
$$

and total differential $D: K^{r} \rightarrow K^{r+1}$ given on $C^{q}\left(\mathcal{U}, \Omega_{X}^{p} \otimes \mathcal{L}^{n-p}\right)$ by $D=\delta+(-l)^{q} i(V)$. Residue formulas involve 0-cocycles in this complex. Note that a total 0-cocycle has the form $\theta=\theta_{0}+\theta_{1}+\cdots+\theta_{n}$, where $\theta_{p} \in C^{p}\left(\mathcal{U}, \Omega_{X}^{p} \otimes \mathcal{L}^{n-p}\right)$ and

$$
\delta \theta_{p}+(-1)^{p} i(V) \theta_{p+1}=0 .
$$

The cocycle condition implies that $\theta_{0} \in C^{0}\left(\mathcal{U}, \mathcal{O}_{X} \otimes \mathcal{L}^{n}\right)$ determines an element $e_{0}(\theta) \in$ $H^{0}\left(Z, \mathcal{L}_{Z}^{n}\right)$. Moreover, $e_{n}(\theta):=\theta_{n} \in C^{n}\left(\mathcal{U}, \Omega_{X}^{n}\right)$ is a cocycle. Let $\boldsymbol{H}^{\bullet}$ denote the cohomology of $K^{\bullet}$. The corresponding morphisms $e_{0}: \boldsymbol{H}^{0} \rightarrow H^{0}\left(Z, \mathcal{L}_{Z}^{n}\right)$ and $e_{n}: \boldsymbol{H}^{0} \rightarrow$ $H^{n}\left(X, \Omega_{X}^{n}\right)$ are canonical edge morphisms in the spectral sequences associated to (3.1), which we will mention again below. 
Proposition 3.3. Let $V \in H^{0}\left(X, \Theta_{X} \otimes \mathcal{L}\right)$ and assume the zero scheme $Z$ of $V$ is non-trivial but isolated. Then (3.1) gives a locally free resolution of $\mathcal{L}_{Z}^{n}$. Moreover, the edge morphism $e_{0}: \boldsymbol{H}^{0} \rightarrow H^{0}\left(Z, \mathcal{L}_{Z}^{n}\right)$ is an isomorphism.

Proposition 3.3 is explicitly proved in [C1]. Now suppose that $\mathcal{E}$ is $V$-equivariant. Then $(2.1)$ says that $\mathcal{V}$ induces an element $V_{Z} \in H^{0}\left(Z,\left(\operatorname{End}_{\mathcal{O}_{X}}(\mathcal{E}) \otimes \mathcal{L}\right)_{Z}\right)$. Thus if $p$ denotes a symmetric $\mathcal{O}_{X}$-linear map $p: \operatorname{End}_{\mathcal{O}_{X}}(\mathcal{E})^{\otimes n} \rightarrow \mathcal{O}_{X}$, then $p\left(V_{Z}\right) \in H^{0}\left(Z, \mathcal{L}_{Z}^{n}\right)$. Thus we define $p_{Z}(E)$ to be $p\left(V_{Z}\right)$ (cf. Section 1). On the other hand, we have already defined $p(E) \in H^{n}\left(X, \Omega_{X}^{n}\right)$. Let $m: H^{0}\left(Z, \mathcal{L}_{Z}^{n}\right) \rightarrow H^{n}\left(X, \Omega_{X}^{n}\right)$ be $e_{n} e_{0}^{-1}$.

Proposition 3.4. $M\left(P\left(v_{z}\right)\right):=M\left(P_{z}(e)\right)=P(e)$.

In the next section, we will establish the residue formula (1.1).

Example (cf. Bott's Theorem). Suppose $V \in H^{0}\left(X, \Theta_{X}\right)$ has simple isolated zeros and $Z$ is non-trivial, say $Z=\left\{\zeta_{1}, \ldots, \zeta_{r}\right\}$. Then, by definition, $\mathcal{O}_{Z, \zeta_{i}}=\mathbb{C}$, and $\mathcal{O}_{Z, x}=0$ if $x \notin Z$. If $E$ is $V$-equivariant, the lift $\mathcal{V}$ of $V$ induces

$$
V_{Z} \in H^{0}\left(Z,\left(\operatorname{End}_{\mathcal{O}_{X}}(\mathcal{E})\right)_{Z}\right)=\bigoplus_{i} \operatorname{End}_{\mathbb{C}}\left(E_{\zeta_{i}}\right)
$$

and

$$
p\left(V_{Z}\right) \in H^{0}\left(Z, \mathcal{O}_{Z}\right)=\bigoplus_{i} \mathbb{C}_{\zeta_{i}}
$$

where $\mathbb{C}_{\zeta_{i}}=\mathbb{C}$ for each $i$. Let $B_{i}:=V_{0, \zeta_{i}} \in \operatorname{End}_{\mathbb{C}}\left(E_{\zeta_{i}}\right)$. Then $p\left(V_{Z}\right)_{\zeta_{i}}=p\left(B_{i}\right)$ for each $i$.

In $[\mathrm{C} 1]$, there is another complex constructed using differential forms in which the map $m$ has a more explicit form.

4. The residue morphism and fundamental commutative diagram. Let $U$ be an open ball in $\mathbb{C}^{n}$ containing the origin 0 , which is the only common zero of $a_{1}, \ldots, a_{n} \in$ $H^{0}(U, \mathcal{L})$. For $s \in H^{0}\left(U, \mathcal{L}^{n} \otimes \Omega_{X}^{n}\right)$ define

$$
\operatorname{Res}\left(\begin{array}{ccc} 
& s \\
a_{1} & \cdots & a_{n}
\end{array}\right)=(2 \pi i)^{-n} \int_{\partial D \times \cdots \times \partial D} \frac{s}{a_{1} \cdots a_{n}},
$$

where $D$ is a disc in $\mathbb{C}$ containing 0 small enough so that $\partial D \times \cdots \times \partial D \cap\left\{a_{1} \cdots a_{n}=\right.$ $0\}=\emptyset$. One easily sees that the right-hand side of (4.1) is independent of such $D$.

Now suppose $\zeta$ is an isolated zero of $V \in H^{0}\left(X, \Theta_{X} \otimes \mathcal{L}\right)$, and let $z_{1}, \ldots, z_{n}$ be local coordinates for $X$ on a neighborhood $U$ of $\zeta$ with $z_{i}(\zeta)=0$ for all $i$. Locally $V=\sum_{i} a_{i} \partial / \partial z_{i}$ where all $a_{i} \in H^{0}(U, \mathcal{L})$. Given $f \in H^{0}\left(U, \mathcal{L}^{n}\right)$, the residue of $f$ with respect to $V$ at $\zeta$ is by definition

$$
\operatorname{Res}_{V, \zeta}(f):=\operatorname{Res}\left(\begin{array}{c}
s \\
a_{1} \cdots a_{n}
\end{array}\right)
$$

where $s=f \otimes d z_{1} \wedge \cdots \wedge d z_{n}$. Again, one can verify $\operatorname{Res}_{V, \zeta}(f)$ is independent of the choices.

The residue has some fundamental properties. First, note that

$$
\frac{\partial a_{i}}{\partial z_{j}}(\zeta)
$$

makes sense, and put

$$
J\left(a_{1}, \ldots, a_{n}\right)_{\zeta}:=\frac{\partial\left(a_{1}, \ldots, a_{n}\right)}{\partial\left(z_{1}, \ldots, z_{n}\right)}(\zeta)
$$


Then

$$
\operatorname{Res}_{V, \zeta}(f)=\frac{f(\zeta)}{J\left(a_{1}, \ldots, a_{n}\right)_{\zeta}},
$$

provided $J\left(a_{1}, \ldots, a_{n}\right)_{\zeta} \neq 0$. Moreover, if $f \in\left(a_{1}, \ldots, a_{n}\right)$, then it is not hard to show that $\operatorname{Res}_{V, \zeta}(f)=0$. It follows that $\operatorname{Res}_{V, \zeta}(f)$ depends only on the element $\tilde{f}(\zeta) \in \mathcal{L}_{Z, \zeta}$ defined by $f$. Therefore, there exists a well defined morphism $\operatorname{Res}_{V}: H^{0}\left(Z, \mathcal{L}_{Z}^{n}\right) \rightarrow \mathbb{C}$ such that $\operatorname{Res}_{V}(\tilde{f})=\sum_{\zeta} \operatorname{Res}_{V, \zeta}(\tilde{f}(\zeta))$. The basic residue theorem is the following

Residue TheOREM. Let $X$ be a compact complex manifold of dimension $n$ and $\mathcal{L}$ an invertible $\mathcal{O}_{X}$-sheaf. Suppose $V \in H^{0}\left(X, \Theta_{X} \otimes \mathcal{L}\right)$ has only isolated zeros, and let $m: H^{0}\left(Z, \mathcal{L}_{Z}^{n}\right) \rightarrow H^{n}\left(X, \Omega_{X}^{n}\right)$ denote the $\mathbb{C}$-linear map defined in Section 3. Then $\operatorname{Res}_{V}:$ $H^{0}\left(Z, \mathcal{L}_{Z}^{n}\right) \rightarrow \mathbb{C}$ can be factored as $\operatorname{Res}_{V}=(-1)^{n} \operatorname{tr} \circ m$, where $\operatorname{tr}: H^{n}\left(X, \Omega^{n}\right) \rightarrow \mathbb{C}$ is $(2 \pi i)^{-n} \int_{X}$. Moreover, suppose $E$ is a $V$-equivariant holomorphic vector bundle on $X$, and $V_{Z}$ denotes the element of $H^{0}\left(Z,\left(\operatorname{End}_{\mathcal{O}_{X}}(\mathcal{E}) \otimes \mathcal{L}\right)_{Z}\right)$ obtained from the lift $\mathcal{V}$ of $V$. Assume $p: \operatorname{End}_{\mathcal{O}_{X}}(\mathcal{E})^{\otimes k} \rightarrow \mathcal{O}_{X}$ is a symmetric $\mathcal{O}_{X}$-linear map. Then

$$
(2 \pi i)^{-n} \int_{X} p(E)=\operatorname{Res}_{V}\left(p\left(V_{Z}\right)\right) \text {. }
$$

In particular, if $k<n$, then $\operatorname{Res}_{V}\left(p\left(V_{Z}\right)\right)=0$.

Proof. The first part of the theorem about $\operatorname{Res}_{V}$ is proved in [C1]. The second assertion is a consequence of the first part, with the use of Proposition 3.4.

By Proposition 2.4, if $X$ is projective algebraic and $E$ is an arbitrary holomorphic vector bundle on $X$, then there exists an invertible sheaf $\mathcal{L}$ and $V \in H^{0}\left(X, \Theta_{X} \otimes \mathcal{L}\right)$ with isolated zeros, so the Residue Theorem is always applicable. More generally, using the formalism of [CL2 §6], this remark also holds for coherent $\mathcal{O}_{X}$-sheaves.

EXAMPLE (continued). Suppose again that $V \in H^{0}\left(X, \Theta_{X}\right)$ has simple isolated zeros $\left\{\zeta_{1}, \ldots, \zeta_{r}\right\}$. Then

$$
A\left(\zeta_{k}\right)=\operatorname{Det}\left(\frac{\partial a_{i}}{\partial z_{j}}\left(\zeta_{k}\right)\right)=J\left(a_{1}, \ldots, a_{n}\right)_{\zeta_{k}} \neq 0 .
$$

Hence Theorem 1 follows immediately from the Residue Theorem and (4.2).

5. Vector fields and cohomology. When $V$ is a holomorphic vector field on a smooth projective variety with zeros, all of which are isolated, the spectral sequences associated to the complex (3.1) when $\mathcal{L}=\mathcal{O}_{X}$ give a striking picture of the ordinary cohomology algebra $H^{*}(X, \mathbb{C})$ of $X$. For brevity, we will refer to [CL1,2] or [C3] for a treatment of the spectral sequences themselves. The upshot is that all $H^{p}\left(X, \Omega_{X}^{q}\right)$ vanish if $p \neq q$, and the coordinate ring $A\left(Z_{V}\right):=H^{0}\left(Z, \mathcal{O}_{Z}\right)$ of the zero scheme $Z$ has a filtration $\mathbb{C}=F_{0} \subseteq F_{1} \subseteq \cdots$ such that $F_{i} F_{j} \subseteq F_{i+j}$, and there are isomorphisms of graded $\mathbb{C}$ algebras $\operatorname{Gr}\left(A\left(Z_{V}\right)\right) \cong \bigoplus_{p} H^{p}\left(X, \Omega_{X}^{p}\right)$, where $\mathrm{Gr}:=\bigoplus_{i \geq 0} F_{i} / F_{i-1}\left(F_{-1}=0\right)$. Because of the Hodge Decomposition Theorem, $H^{r}(X, \mathbb{C})=\bigoplus_{p+q=r} H^{p}\left(X, \Omega_{X}^{q}\right)$, it follows that $\operatorname{Gr}\left(A\left(Z_{V}\right)\right) \cong H^{*}(X, \mathbb{C})$. Note that the isomorphism doubles degrees.

When $V$ is generated by a $\mathbb{C}^{*}$-action and all $H^{p}\left(X, \Omega_{X}^{q}\right)$ vanish if $p \neq q$, then one has the following result in which $Z$ can have positive dimension.

TheOREM $5.1([\mathrm{C} 2])$. Assume $X$ is a projective variety such that all $H^{p}\left(X, \Omega_{X}^{q}\right)$ vanish if $p \neq q$, and let $Z$ be the (necessarily non-empty) fixed point set of a $\mathbb{C}^{*}$-action on $X$. 
Then there exists a filtration of the cohomology algebra $H^{*}(Z, \mathbb{C})$ whose associated graded algebra is $H^{*}(X, \mathbb{C})$.

This result was applied in [C2] to study cohomology of Springer fibers in a $G / B$. There are many situations in which $Z$ has positive dimension, yet the filtration $F$. of the algebra $H^{*}(Z, \mathbb{C})$ is little understood, and it seems to be important to obtain a better understanding of the situation. Recently, the filtration has been described when the $\mathbb{C}^{*}$-action is embedded as a maximal torus of a connected two-dimensional solvable group $\mathcal{B}$ acting on $X$ with a unique fixed point [C5]. In the rest of the paper, we describe this result.

Let $\mathcal{B}$ denote the upper triangular Borel subgroup of $S L_{2}(\mathbb{C})$ with standard $\left(G_{m}, G_{a}\right)$ pair $(\lambda, \phi)$ satisfying

$$
\lambda(a) \phi(t) \lambda(a)^{-1}=\phi\left(a^{2} t\right)
$$

for all $a \in \mathbb{C}^{*}, t \in \mathbb{C}$. Here $\lambda(a)=\operatorname{diag}\left[a, a^{-1}\right]$ and

$$
\phi(t)=\left(\begin{array}{ll}
1 & t \\
0 & 1
\end{array}\right) .
$$

$X$ will denote a smooth projective variety with an algebraic $\mathcal{B}$-action $(b, x) \rightarrow b \cdot x$ having a unique $\mathcal{B}$-fixed point $o . X^{\lambda}$ and $X^{\phi}$ will denote respectively the fixed point sets of the $G_{m^{-}}$and $G_{a^{-}}$-actions on $X$ induced by $\lambda$ and $\phi$. It is known that in this situation $X^{\lambda}$ is finite and $X^{\phi}=\{o\}$, so we may write $X^{\lambda}=\left\{x_{1}, \ldots, x_{r}\right\}$. Note also that $o \in X^{\lambda}$. Recall that the Bialynicki-Birula plus and minus cells associated to $\lambda$ are respectively

$$
x_{i}^{+}:=\left\{x \in X \mid \lim _{a \rightarrow 0} \lambda(a) \cdot x=x_{i}\right\}
$$

and

$$
x_{i}^{-}:=\left\{x \in X \mid \lim _{a \rightarrow \infty} \lambda(a) \cdot x=x_{i}\right\} .
$$

By $[\mathrm{BB}]$, the plus and minus cells define two locally closed $\lambda$-stable decompositions of $X$ into affine spaces. A basic fact is that $U:=o^{-}$is the open minus cell (the so called big cell). Hence, there exist affine coordinates $w_{1}, \ldots, w_{n}$ on $U$ which are homogeneous with respect to the $\mathbb{C}^{*}$-action $\lambda$ and in fact, have positive even degrees. Consequently, the coordinate ring $A(U)$ is the graded $\mathbb{C}$-algebra $\mathbb{C}\left[w_{1}, \ldots, w_{n}\right]$, which is concentrated in even positive degrees. This grading is called the principal grading.

Let $(V, W)$ denote the pair of algebraic vector fields on $X$ generated by $(\phi, \lambda) . W$ has simple isolated zeros $\left\{x_{1}, \ldots, x_{r}\right\}$, while $V$ has a single non-reduced zero at $o$. The basic deformation of $(V, W)$ is the family of vector fields $\left\{V^{s}=V+s W \mid s \in \mathbb{C}\right\}$ on $X$.

Proposition 5.2 ([C5]). Assume $\mathcal{B}$ acts algebraically on $X$ with exactly one fixed point. Then for $s \neq 0, V^{s}$ has $\chi(X)$ simple isolated zeros all of which lie in the big cell.

We will denote the zero scheme of $V=V^{0}$, which is supported by $o$, by $Z$ instead of $Z^{0}$. By Proposition 5.2, if $s \neq 0$, the zero scheme $Z^{s}$ of $V^{s}$ is reduced. The intuitive picture is that for $s \neq 0, Z^{s}$ is a transverse splitting of the zero $o$ of $V$. By the general theory outlined above, the coordinate ring $A\left(Z^{s}\right)$ has a filtration $F^{s}$ whose associated graded is the cohomology of $X$. It turns out that in our setting, the filtrations have natural descriptions, although the proof of this is somewhat complicated. To describe these filtrations, note that since $Z^{s} \subset U$ for all $s \in \mathbb{C}$, the coordinate $\operatorname{ring} A\left(Z^{s}\right)=H^{0}\left(Z^{s}, \mathcal{O}_{Z^{s}}\right)$ is the quotient of $A(U)=\mathbb{C}\left[w_{1}, \ldots, w_{n}\right]$ by the ideal $I_{Z^{s}}$ generated by $V^{s}\left(w_{1}\right), \ldots, V^{s}\left(w_{n}\right)$. Thus there 
is a natural principal filtration $P^{s}$ of $A\left(Z^{s}\right)$ satisfying $P_{i}^{s} P_{j}^{s} \subseteq P_{i+j}^{s}$. Namely, we define $P_{i}^{s}:=\operatorname{Im}\left[\bigoplus_{j \leq i} A(U)_{2 j} \rightarrow A\left(Z^{s}\right)\right]$.

Theorem 5.3. For each $s \in \mathbb{C}$, the filtrations $F^{s}$ and $P^{s}$ of $A\left(Z^{s}\right)$ coincide. Hence there is a graded $\mathbb{C}$-algebra isomorphism $\Phi_{s}: \operatorname{Gr}_{P} A\left(Z^{s}\right) \rightarrow H^{*}(X)$. Moreover, the ideal defining $A(Z)$ is homogeneous, so $\Phi_{0}: A(Z) \rightarrow H^{*}(X)$ is an isomorphism of graded $\mathbb{C}$-algebras.

The part of Theorem 5.3 dealing with $\Phi_{0}$ goes back to [ACLS] and is proved in [AC1]. The proof for $s \neq 0$ will appear in [C5]. In [AC2], it was noticed that the presentation of $H^{*}(X, \mathbb{C})$ as $A(Z)$ gives a product formula for the Poincaré polynomial of $X$.

In the remainder of this section, we will outline some recent results on cohomology of (possibly) singular $\mathcal{B}$-stable subvarieties of $X$. The first step is to glue the zero sets of the $V^{s}$ in $U \times \mathbb{C}$ to form a curve $\mathcal{Z}=\left\{(x, s) \mid V^{s}(x)=0\right\}$. $\mathcal{Z}$ has $\chi(X)$ irreducible components, and any two components meet exactly at $(o, 0)$. The interesting point seems to be the nature of the singularity of $\mathcal{Z}$ at this point. If $Y$ is a $\mathcal{B}$-stable subvariety of $X$, put $\mathcal{Z}_{Y}:=\mathcal{Z} \cap(Y \times \mathbb{C})$. Thus $\mathcal{Z}_{Y}$ is the union of the irreducible components of $\mathcal{Z}$ which meet $Y \times \mathbb{C}^{*}$.

TheOREM 5.4 ([C5]). Suppose $Y$ is a $\mathcal{B}$-stable subvariety of $X$ and that the cohomology restriction map $i_{Y}^{*}: H^{*}(X, \mathbb{C}) \rightarrow H^{*}(Y, \mathbb{C})$ is surjective. Let $\mathcal{R}_{Y}$ denote the coordinate ring of the schematic intersection of $\mathcal{Z}_{Y}$ and $U \times 0$ in $U \times \mathbb{C}$. Then $\mathcal{R}_{Y}$ has a natural grading and there exists an isomorphism of graded rings $\Psi_{Y}: \mathcal{R}_{Y} \rightarrow H^{*}(Y, \mathbb{C})$ which is natural with respect to restrictions.

The last assertion means that if $W$ is a $\mathcal{B}$-stable subvariety of $Y$ such that $i_{W}^{*}$ : $H^{*}(X, \mathbb{C}) \rightarrow H^{*}(W, \mathbb{C})$ is surjective, then $i^{*} \Psi_{Y}=\Psi_{W} j^{*}$, where $i^{*}: H^{*}(Y, \mathbb{C}) \rightarrow$ $H^{*}(W, \mathbb{C})$ and $j^{*}: \mathcal{R}_{Y} \rightarrow \mathcal{R}_{W}$ are the restriction maps.

The surjectivity assumption is necessary in order that $\Psi_{Y}$ be an isomorphism, since $\Psi_{Y}$ cannot be surjective unless $i_{Y}$ is. In fact, surjectivity holds in a fairly general situation. Suppose $X$ is a projective spherical $B$-variety such that $X^{B}$ is a single point ( $B$ is here a Borel subgroup in a semisimple algebraic group). Suppose furthermore that every $B$-orbit is a product of $\mathbb{C}$ 's. That is, the $B$-orbits do not have $\mathbb{C}^{*}$ factors. Then it is a result of DeConcini and Springer [DS] that the $B$-orbits are actually plus cells, for a suitable $G_{m}$-action on $X$ (arising from a maximal torus in $B$ ). That being the case, it is obvious that if $Y$ is the closure of a $B$-orbit, then $i_{Y}^{*}$ is surjective. Thus Theorem 5.4 applies as long as there exists a $\mathcal{B} \subseteq B$ for which $X^{\mathcal{B}}$ is a single point. (I am indebted to Michel Brion for pointing out that this may not always be the case.) In particular, these remarks apply when $X$ is an algebraic homogeneous space $G / P$, where $G$ is a linear algebraic group over $\mathbb{C}$ and $P$ is a parabolic subgroup.

The following result shows that sometimes it is possible to describe $H^{*}(Y, \mathbb{C})$ in terms of $Z$ and the ideal of the affine variety $Y^{o}=Y \cap U$. Indeed, let $A(Z \cap Y)$ denote the coordinate $\operatorname{ring} A(U) /\left(I(Z)+I\left(Y^{o}\right)\right)$ of the scheme theoretic intersection of $Z$ and $Y^{o}$. Then we have

TheOREm 5.5. Suppose $Y$ is a $\mathcal{B}$-stable subvariety of $X$ and the cohomology restriction map $i_{Y}^{*}: H^{*}(X, \mathbb{C}) \rightarrow H^{*}(Y, \mathbb{C})$ is surjective. Then there exists an isomorphism of graded rings $\Phi_{Y}: A(Z \cap Y) \rightarrow H^{*}(Y, \mathbb{C})$ if and only if the scheme theoretic and variety theoretic intersections of $\mathcal{Z}$ and $Y^{o} \times 0$ in $X \times \mathbb{C}$ coincide. 
The condition that the scheme theoretic and variety theoretic intersections of $\mathcal{Z}$ and $Y^{o} \times 0$ in $X \times \mathbb{C}$ coincide is simply that $I\left(\mathcal{Z}_{Y}\right)=I(\mathcal{Z})+I\left(Y^{o} \times \mathbb{C}\right)$. The map $\Phi_{Y}$ was studied in the case of Schubert varieties in a Grassmannian by E. and Y. Akyildiz in [AA] and by E. Akyildiz, A. Lascoux and P. Pragacz [ALP] when $Y$ is a Schubert subvariety in the variety of complete flags in $\mathbb{C}^{n}$. In both of these cases it was shown that $\Phi_{Y}$ is always a graded ring isomorphism. However, there are examples due to Dale Peterson (see the appendix of [C4]) where $X$ is $G / B$ with $G \neq S L(n, \mathbb{C})$ and $Y$ is a Schubert variety for which $A(Z \cap Y)$ and $H^{*}(Y, \mathbb{C})$ have different dimensions over $\mathbb{C}$.

Assuming that $i_{Y}^{*}$ is surjective, it is not hard to deduce that the curve $\mathcal{Z}_{Y}$ is Gorenstein if and only if $Y$ is a rational homology manifold. Recently the author has shown that under certain (probably unnecessary) mild restrictions, $Y$ is non-singular if and only if $\mathcal{Z}_{Y}$ is a locally complete intersection.

\section{References}

[AA] E. Akyildiz and Y. Akyildiz, The relations of Plücker coordinates to Schubert calculus, J. Differential Geom. 29 (1989), 135-142.

[AC1] E. Akyildiz and J. B. Carrell, Cohomology of projective varieties with regular $S L_{2}$ actions, Manuscripta Math. 58 (1987), 473-486.

[AC2] E. Akyildiz and J. B. Carrell, A generalization of the Kostant-Macdonald identity, Proc. Nat. Acad. Sci. U.S.A. 86 (1989), 3934-3937.

[ACLS] E. Akyildiz, J. B. Carrell, D. I. Lieberman and A. J. Sommese, On the graded rings associated to holomorphic vector fields with exactly one zero, Proc. Sympos. Pure Math. 40 (1983), 55-57.

[ALP] E. Akyildiz, A. Lascoux and P. Pragacz, Cohomology of Schubert subvarieties of $G L_{n} / P$, J. Differential Geom. 35 (1992), 511-519.

[At] M. F. Atiyah, Complex analytic connections in fibre bundles, Trans. Amer. Math. Soc. 85 (1957), 181-207.

[Be] A. Beauville, Une notion de résidu en géométrie analytique, in: Séminaire Pierre Lelong (Analyse). Année 1970. Lecture Notes in Math. 205, Springer-Verlag, Berlin and New York, 1971, 183-203.

[BB] A. Białynicki-Birula, Some theorems on actions of algebraic groups, Ann. of Math. (2) 98 (1973), 480-497.

[B1] R. Bott, Vector fields and characteristic numbers, Michigan Math. J. 40 (1967), 231244.

[B2] R. Bott, A residue formula for holomorphic vector fields, J. Differential Geom. 1 (1967), 311-330.

[B3] R. Bott, On a topological obstruction to integrability, Proc. Sympos. Pure Math. 16 (1970), 127-132.

[C1] J. B. Carrell, A remark on the Grothendieck residue map, Proc. Amer. Math. Soc. 70 (1978), 43-48.

[C2] J. B. Carrell, Orbits of the Weyl group and a theorem of DeConcini and Procesi, Compositio Math. 60 (1986), 45-52.

[C3] J. B. Carrell, Vector fields, flag varieties and Schubert calculus, in: Proceedings of the Hyderabad Conference on Algebraic Groups, Manoj-Prakashan, Madras, 1991, 23-59.

[C4] J. B. Carrell, Bruhat cells in the nilpotent variety and the intersection rings of Schubert varieties, J. Differential Geom. 37 (1993), 651-668.

[C5] J. B. Carrell, Deformation of the nilpotent zero scheme and the intersection ring of invariant subvarieties, to appear in J. Reine Angew. Math. 
[CL1] J. B. Carrell, and D. I. Lieberman, Holomorphic vector fields and compact Kaehler manifolds, Invent. Math. 21 (1973), 303-309.

[CL2] J. B. Carrell and D. I. Lieberman, Vector fields and Chern numbers, Math. Ann. 225 (1977), 263-272.

[DS] C. DeConcini and T. A. Springer, Betti numbers of complete symmetric varieties, Geometry Today, Birkhäuser (1985).

[H] R. Hartshorne, Residues and duality, Lecture Notes in Math. 20, Springer-Verlag, Berlin and New York, 1966.

[L] J. Lipman, Dualizing sheaves, differentials and residues on algebraic varieties, Astérisque 117 (1984).

[V] J. L. Verdier, Base change for twisted inverse image of coherent sheaves, in: Algebraic Geometry, Oxford University Press, London, 1969, 393-408. 